\title{
An absolute quantum energy inequality for the Dirac field in curved spacetime
}

\author{
Calvin J. Smith* \\ School of Mathematical Sciences, University College Dublin, \\ Belfield, Dublin 4, Ireland
}

15 May 2007

\begin{abstract}
Quantum Weak Energy Inequalities (QWEIs) are results which limit the extent to which the smeared renormalised energy density of a quantum field can be negative. On globally hyperbolic spacetimes the massive quantum Dirac field is known to obey a QWEI in terms of a reference state chosen arbitrarily from the class of Hadamard states; however, there exist spacetimes of interest on which state-dependent bounds cannot be evaluated. In this paper we prove the first QWEI for the massive quantum Dirac field on four dimensional globally hyperbolic spacetime in which the bound depends only on the local geometry; such a QWEI is known as an absolute QWEI.
\end{abstract}

\section{Introduction}

When formulating the classical theory of general relativity it is necessary to impose certain energy conditions on the source matter fields being considered. The most commonplace of these energy conditions is the weak energy condition, $T_{a b} k^{a} k^{b} \geq 0$ for every timelike vector field $k$, which entails that observers only encounter positive energy densities. However, it has been known since 1965 that, unlike most classical physics models, no (Wightman) quantum field theory can obey pointwise energy conditions [5]. Moreover, it is possible to show that the negative energy density arising from a quantum field theoretic source is unbounded (from below) in magnitude [10]. This startling feature of quantum field theory is often used, in the context of the semi-classical Einstein equation $G_{a b}=8 \pi G\left\langle T_{a b}^{\mathrm{ren}}\right\rangle_{\omega}$, to support so-called 'designer spacetimes' like Alcubierre's warp drive [1] or traversable worm hole geometries. Following Ford's [13] observation that it is

*Electronic address: calvin.smith@ucd.ie 
possible to bound the magnitude and duration of the flux of negative energy of a quantum field source, work began in earnest to prove that the averaged (expectation value of the) energy density of a quantum field was bounded from below. A suitable definition, sufficient for our purposes 1 , is that a worldline quantum weak energy inequality (QWEI) is a result of the form

$$
\int_{\mathbb{R}} \mathrm{d} \tau\left\langle\rho^{\mathrm{ren}}\right\rangle_{\omega}(\gamma(\tau)) F(\tau) \geq-\mathcal{B}>-\infty
$$

where $F$ is some appropriately chosen sampling function, $\gamma: \mathbb{R} \mapsto \mathcal{M}$ is a timelike worldline and $\left\langle\rho^{\mathrm{ren}}\right\rangle_{\omega}$ is the (expectation value of the) energy density of the quantum field in a state $\omega$. In this discussion we shall exclusively consider the massive quantum Dirac field in a smooth four-dimensional globally hyperbolic spacetime $(\mathcal{M}, g)$. Moreover, we shall only consider the Hadamard states of the Dirac field as this is a sufficient class of states to renormalise the stress energy density.

Typically, the bound $\mathcal{B}$ featuring in (11) is a function of another state of the theory usually called a reference state; these QWEIs are known as difference QWEIs. Due to the work of Fewster and his collaborators [3, 7], difference QWEIs are known in great generality for the Dirac field in curved spacetime. (For a brief review of QWEIs for other fields the reader is directed to section one of [12] and the references therein). Difference QWEIs have been instrumental in constraining the likelihood of designer spacetime manifestation; however, there exist spacetimes on which one does not know how to write down the closed form expressions for states necessary for the evaluation of the difference QWEI bound. Indeed, the warp drive is an example of a spacetime on which it is not currently known how to obtain explicit expressions for Hadamard states. Therefore, it is desirable to have a lower bound $\mathcal{B}$ which is state independent and constructed only from the local geometry; such a bound is known as an absolute QWEI. Currently, for the Dirac field, absolute QWEIs are known only for the conformally invariant [26] and massive field in two-dimensions [2] and the massive field in four-dimensional flat spacetime [8]. In this discussion we state and prove the first absolute QWEI for the massive Dirac field in arbitrary four-dimensional globally hyperbolic spacetime. The argument is an adaptation of Fewster's earlier work with Verch [7] and Dawson [3] and is to be viewed as a companion to the analogous result for the Klein-Gordon field [12].

Our result may be stated as follows: Let $(\mathcal{M}, g)$ be a classical curved four-dimensional spacetime. Here $\mathcal{M}$ is a four-dimensional smooth manifold (assumed Hausdorff, paracompact and without boundary) with a Lorentz metric $g_{a b}$ of signature $(+---)$. Furthermore, we require $(\mathcal{M}, g)$ to be globally hyperbolic, that is $\mathcal{M}$ contains a Cauchy surface. In addition we assume that an orientation, time orientation and spin structure have been chosen. It may be shown that on such a background one may formulate the quantum Dirac field and a notion of Hadamard states. The essential feature of Hadamard states is that they all share a common singularity structure; in particular their two-point functions, and their Dirac adjoints, have a local and covariantly determined singular expansion. We denote the Hadamard series corresponding to the singularity structure of

\footnotetext{
${ }^{1} \mathrm{~A}$ more general, and rigorous, definition of a quantum energy inequality is given in [11].
} 
the Dirac two-point function $\mathcal{W}_{\omega}$ by ${ }^{\psi} H_{k}^{(+)}$and that corresponding to the singular structure of the adjoint Dirac two-point function $\mathcal{W}_{\omega}^{\Gamma}$ by ${ }^{\psi} H_{k}^{(-)}$. The salient feature of such states is that one may define a finite stress energy density $\left\langle\rho^{\mathrm{fin}}\right\rangle_{\omega}(x)$ by using $\mathcal{W}_{\omega}-{ }^{\psi} H_{k}^{(+)}$ and a point-splitting prescription. Indeed, define an operator $\rho^{\text {split }}$ such that the finite contribution to the energy density is given by $\rho^{\text {split }}$ acting on the regularised two-point function of the Dirac field $\mathcal{W}_{\omega}-{ }^{\psi} H_{k}^{(+)}$; i.e.

$$
\left\langle\rho^{\mathrm{fin}}\right\rangle_{\omega}(x):=\lim _{x^{\prime} \rightarrow x}\left[\rho^{\mathrm{split}}\left(\mathcal{W}_{\omega}-{ }^{\psi} H_{1}^{(+)}\right)\right]\left(x, x^{\prime}\right) .
$$

The precise form of $\rho^{\text {split }}$ is given in 92.4 . The quantity $\left\langle\rho^{\text {fin }}\right\rangle_{\omega}$ is equal to the renormalised energy density modulo a local curvature term; we shall return to this issue later after proving our main result in theorem 5.1. Our result then reads for any real valued $f \in C_{0}^{\infty}(\mathbb{R})$ and Hadamard state $\omega$

$$
\int_{\mathbb{R}} \mathrm{d} \tau\left\langle\rho^{\mathrm{ren}}\right\rangle_{\omega}(\gamma(\tau)) f^{2}(\tau) \geq-\mathcal{B}
$$

modulo local curvature terms, where $\mathcal{B}$ is of the form

$$
\begin{aligned}
\mathcal{B}= & \int_{\mathbb{R}^{+}} \frac{\mathrm{d} \xi}{2 \pi} \xi\left[f \otimes f \vartheta^{*} \psi \mathrm{H}_{4}^{(+)}\right]^{\wedge}(-\xi, \xi) \\
& -\int_{\mathbb{R}^{-}} \frac{\mathrm{d} \xi}{2 \pi} \xi\left[f \otimes f \vartheta^{*}\left(i \mathrm{~S}_{\mathrm{sp}}-{ }^{\psi} \mathrm{H}_{4}^{(+)}\right)\right]^{\wedge}(-\xi, \xi) .
\end{aligned}
$$

Here $\vartheta=\gamma \otimes \gamma, S_{\mathrm{sp}}$ is the fundamental solution to the Dirac equation, ${ }^{\psi} \mathrm{H}_{k}^{( \pm)}$are scalar distributions created from ${ }^{\psi} H_{k}^{( \pm)}$and ^ denotes the Fourier transform which in our conventions is given by

$$
\hat{f}(\xi)=\int \mathrm{d} x f(x) e^{i \xi \cdot x}
$$

The structure of this paper is as follows: In section 2 we present a review of the formulation of the classical $(\$ 2.1+2.2)$ and quantum (\$2.3) Dirac fields and their Hadamard states. We then direct our attention to a microlocal description of the Hadamard series for the Dirac field in section 3, in particular we review the Sobolev wave-front set and its properties (33.1) before applying the theory to the matter in hand and obtaining estimates on the singularities of the Hadamard series (\$3.2). Finally, a point-splitting lemma is presented in section 4 before our main result is stated in section 5 .

\section{The Dirac field in curved spacetime}

The reader who is familiar with the formalism necessary to describe the classical Dirac field on a curved background is encouraged to skip ahead to section $\$ 2.3$. 


\subsection{Spin structures and spinors on curved spacetimes}

We begin by reviewing the geometry necessary to discuss the Dirac field in a curved spacetime. We shall employ the algebraic framework for describing the Dirac quantum field in a classical curved four-dimensional spacetime $(\mathcal{M}, g)$. Here $\mathcal{M}$ is a fourdimensional smooth manifold (assumed Hausdorff, paracompact and without boundary) with a Lorentz metric $g_{a b}$ of signature $(+---)$. Furthermore, we require $(\mathcal{M}, g)$ to be globally hyperbolic, that is $\mathcal{M}$ contains a Cauchy surface. Where index notation is used, Latin indices will run over the range $0,1,2,3$ unless explicitly stated otherwise, while Greek characters will denote frame indices and also run over 0,1,2,3 unless explicitly stated otherwise. We employ units in which $c=\hbar=1$.

In Minkowski spacetime the spinors are nothing more than the spin-half representation of the Poincaré group, however, a general manifold does not exhibit this symmetry globally: Therefore, the usual (i.e. Minkowski spacetime) interpretation of a spinor as being a quadruple of complex numbers at each point in spacetime does not generalise under the replacement $\left(\mathbb{R}^{4}, \eta\right) \mapsto(\mathcal{M}, g)$. A rigorous formulation of spinors on a manifold is given in terms of fibre bundles where the spin group is the structure group. (For a review of the necessary concepts related to fibre bundles, and in particular spinor bundles, the reader is directed to [21]). We shall review basic facts about the Dirac matrices and the Lorentz and spin groups in Minkowski spacetime, and use a local frame to generalise the results to a curved spacetime. What follows is based on [3] and benefits from the elaborations in [4, 7].

We begin by summarising several groups which appear in our discussion. The Lorentz group $O(1,3)=\left\{\Lambda \in \mathrm{GL}_{4}(\mathbb{R}) \mid \eta_{\alpha \beta} \Lambda_{\gamma}^{\alpha} \Lambda_{\delta}^{\beta}=\eta_{\gamma \delta}\right\}$ has the subgroup $\mathfrak{L}_{+}^{\uparrow}$,

$$
\mathfrak{L}_{+}^{\uparrow}=\left\{\Lambda \in O(1,3) \mid \operatorname{det} \Lambda=1 \& \Lambda_{0}^{0}>0\right\}
$$

called the proper orthochronous Lorentz group. The Dirac gamma matrices $\gamma_{\alpha}$ satisfy the Clifford algebra relation $\left\{\gamma_{\alpha}, \gamma_{\beta}\right\}=2 \eta_{\alpha \beta} \mathbb{1}$ and are said to belong to a standard representation if $\gamma_{0}^{\dagger}=\gamma_{0}$ and $\gamma_{\alpha}^{\dagger}=-\gamma_{\alpha}$ for $\alpha=1,2,3$. From here on we shall assume that our Dirac matrices belong to a standard representation. The spin group, $\operatorname{Spin}(1,3)$, is defined by

$$
\operatorname{Spin}(1,3)=\left\{S \in \mathrm{SL}_{4}(\mathbb{C}) \mid S \gamma_{\alpha} S^{-1}=\gamma_{\beta} \Lambda_{\alpha}^{\beta} \text { for some } \Lambda \in \mathcal{L}\right\}
$$

and is known to be a two-to-one cover of $\mathfrak{L}_{+}^{\uparrow}$, i.e. the mapping $S \mapsto \Lambda(S)$ is a twoto-one covering homomorphism from the identity connected component $\operatorname{Spin}_{0}(1,3)$, of $\operatorname{Spin}(1,3)$, to $\mathfrak{L}_{+}^{\uparrow}$ with kernel $\{\mathbb{1},-\mathbb{1}\}$.

We now direct our attention to a curved spacetime setting. The frame bundle $F \mathcal{M}$ is the bundle of oriented and time-oriented tetrads $\left\{e_{\alpha}^{a}\right\}_{\alpha=0,1,2,3}$ over spacetime $(\mathcal{M}, g)$ with the convention that $e_{0}^{a}$ is a future pointing timelike vector; moreover, $F \mathcal{M}$ is a principal $\mathcal{L}_{+}^{\uparrow}$ bundle whose right action is given by $\left(R_{\Lambda} e\right)_{\alpha}=e_{\beta} \Lambda^{\beta}{ }_{\alpha}$. A spin structure on $(\mathcal{M}, g)$ is a principal $\operatorname{Spin}_{0}(1,3)$ bundle, $S \mathcal{M}$, over $(\mathcal{M}, g)$ equipped with a fibre homomorphism $\varphi: S \mathcal{M} \mapsto F \mathcal{M}$ such that $\varphi \circ R_{S}=R_{\Lambda(S)} \circ \varphi$, i.e. $\varphi$ intertwines the 
right action of the structure group on these bundles. Spin structures are not unique, however two such structures, $S \mathcal{M}$ and $\widetilde{S \mathcal{M}}$ equipped with $\varphi$ and $\widetilde{\varphi}$ respectively can be said to be equivalent if there is an isomorphism $\iota: S \mathcal{M} \mapsto \widetilde{S \mathcal{M}}$ such that $\varphi=\widetilde{\varphi} \circ \iota$.

It is worth pointing out that spin structures do not exist in general for an arbitrary manifold; their existence is determined by the second Stiefel-Whitney class. In essence, the requirement that the second Stiefel-Whitney class vanishes ensures consistency between the (transition functions of the) fibre group of the tangent bundle and the (lift to the transition functions of the) spin group. It is known that there exist spin structures over orientable manifolds if and only if the second Stiefel-Whitney class vanishes and that every four-dimensional globally hyperbolic manifold admits a spin structure. We now assume that an arbitrary spin structure has been chosen and is fixed for the remainder of this discussion.

We may now define spinor fields on a curved manifold by saying they are sections of the associated $\operatorname{Spin}_{0}(1,3)$ bundle

$$
D \mathcal{M}=S \mathcal{M} \ltimes_{\operatorname{Spin}_{0}(1,3)} \mathbb{C}^{4} .
$$

The fibre of $D \mathcal{M}$ at $x \in \mathcal{M}$ is the equivalence class $[T, z]_{x}$ where $T \in S_{x} \mathcal{M}$ and $z \in \mathbb{C}^{4}$ is a column vector and the equivalence relation is: $[\widetilde{T}, \widetilde{z}]_{x}=[T, z]_{x}$ if and only if $\widetilde{T}=R_{S}^{-1} T$ and $\widetilde{z}=S z$ for some $S \in \operatorname{Spin}_{0}(1,3)$. The bundle $D \mathcal{M}$ has fibre $\mathbb{C}^{4}$ at every point and left action given by $L_{S}[T, z]_{x}=[T, S z]_{x}$. The dual bundle

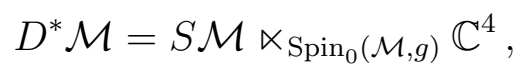

where $\mathbb{C}^{4}$ is the set of complex row 4 -vectors, is constructed similarly and its fibres are the equivalence classes $\left[T, z^{\mathrm{t}}\right]_{x}^{*}, z^{\mathrm{t}} \in \mathbb{C}^{4}$ a row vector, such that $\left[\widetilde{T}, \widetilde{z^{\mathrm{t}}}\right]_{x}^{*}=\left[T, z^{\mathrm{t}}\right]_{x}^{*}$ if and only if $\widetilde{T}=R_{S}^{-1} T$ and $\widetilde{z^{\mathrm{t}}}=z^{\mathrm{t}} S^{-1}$ for some $S \in \operatorname{Spin}_{0}(1,3)$. Just as the sections of $D \mathcal{M}$ are called spinors, the sections of $D^{*} \mathcal{M}$ are called cospinors. We shall refer to test spinors as being the smooth and compactly supported sections of $D \mathcal{M}$, the space of which we denote $C_{0}^{\infty}(D \mathcal{M})$; test cospinors are similarly defined and are elements of $C_{0}^{\infty}\left(D^{*} \mathcal{M}\right)$. As expected, there exists a natural pairing between spinors and cospinors: Set $v_{x}=\left[T, z_{1}^{\mathrm{t}}\right]_{x}^{*}$ and $u_{x} \in\left[T, z_{2}\right]_{x}$, then $v_{x}\left(u_{x}\right)=z_{1}^{\mathrm{t}} z_{2}$ is a scalar.

We are now in a position to define the Dirac adjoint operation ${ }^{+}: D \mathcal{M} \mapsto D^{*} \mathcal{M}$ which is given by

$$
[T, z]_{x}^{+}=\left[T, z^{\dagger} \gamma_{0}\right]_{x}^{*} .
$$

Any local section $E: \mathcal{M} \mapsto S \mathcal{M}$ of $S \mathcal{M}$ determines a local frame $e_{\alpha}^{a}$ by $\varphi \circ E$ and local sections $E_{A}$ of $D \mathcal{M}$, such that $E_{A}(x)=\left[E_{x}, z_{A}\right]$ where $\left\{z_{A}\right\}_{A=0,1,2,3}$ is the canonical basis of $\mathbb{C}^{4}$. The dual frames $e_{a}^{\alpha}, E^{A}$ are defined through $e^{\alpha} \cdot e_{\beta}=\delta_{\beta}^{\alpha}$ and $E^{A}\left(E_{B}\right)=\delta_{B}^{A}$. One may define a mixed tensor-spinor object $\gamma \in C^{\infty}\left(T^{*} \mathcal{M}\right) \otimes C^{\infty}(D \mathcal{M}) \otimes C^{\infty}\left(D^{*} \mathcal{M}\right)$ by setting its components $\gamma_{\alpha}{ }^{A}{ }_{B}$ in the frame $e_{a}^{\alpha} \otimes E_{A} \otimes E^{B}$ equal to the matrix elements $\left(\gamma_{\alpha}\right)^{A}$. For example, it can be shown that

$$
\gamma_{0}=\delta^{A B} E_{A} \otimes E_{B}^{+} .
$$




\subsection{The Dirac equation}

The metric $g$ determines a connection $\Gamma$ in the usual way via the covariant derivative operator $\nabla: C^{\infty}(T \mathcal{M}) \mapsto C^{\infty}\left(T^{*} \mathcal{M} \otimes T \mathcal{M}\right)$. One may equally define a connection $\sigma$ and covariant derivative on the spinor and cospinor bundle, which we also denote by $\nabla$,

$$
\nabla:\left\{\begin{array} { c } 
{ C ^ { \infty } ( D \mathcal { M } ) } \\
{ C ^ { \infty } ( D ^ { * } \mathcal { M } ) }
\end{array} \mapsto \left\{\begin{array}{c}
C^{\infty}\left(T^{*} \mathcal{M} \otimes D \mathcal{M}\right) \\
C^{\infty}\left(T^{*} \mathcal{M} \otimes D^{*} \mathcal{M}\right)
\end{array} .\right.\right.
$$

Given a local section $E, f \in C^{\infty}(D \mathcal{M})$ may be decomposed $f=f^{A} E_{A}$, then $\nabla_{a} f$ has components

$$
\nabla_{\alpha} f^{A}=\partial_{\alpha} f^{A}+\sigma_{\alpha B}^{A} f^{B}
$$

in the frame $e_{a}^{\alpha} \otimes E_{A}$ where the connection $\sigma$ has elements given by $\sigma_{\alpha B}^{A}=-\frac{1}{4} \Gamma_{\alpha \delta}^{\beta} \gamma_{\beta}^{A}{ }_{C} \gamma^{\delta C}$.

We are now in a position to define the equation of motion the spinors will satisfy, i.e. the Dirac equation. The Dirac operator

$$
\not:\left\{\begin{array} { l } 
{ C ^ { \infty } ( D \mathcal { M } ) } \\
{ C ^ { \infty } ( D ^ { * } \mathcal { M } ) }
\end{array} \mapsto \left\{\begin{array}{l}
C^{\infty}(D \mathcal{M}) \\
C^{\infty}\left(D^{*} \mathcal{M}\right)
\end{array}\right.\right.
$$

maps (co)spinor fields into (co)spinor fields by

$$
\begin{array}{lll}
\not \nabla f & =(\not \nabla f)^{A} E_{A}=\eta^{\alpha \beta} \gamma_{\alpha}{ }^{A}{ }_{B}\left(\nabla_{\beta} f^{B}\right) E_{A} & \forall f \in C^{\infty}(D \mathcal{M}) \\
\not h & =(\not h h)_{B} E^{B}=\eta^{\alpha \beta}\left(\nabla_{\beta} h_{C}\right) \gamma_{\alpha}{ }^{C}{ }_{B} E^{B} & \forall h \in C^{\infty}\left(D^{*} \mathcal{M}\right) .
\end{array}
$$

The spinor field $f \in C^{\infty}(D \mathcal{M})$ is said to satisfy the Dirac equation if $(-i \not \nabla+\mu) f=0$ where the constant $\mu \geq 0$ is interpreted as the mass of the field. Similarly, the cospinor field $h \in C^{\infty}\left(D^{*} \mathcal{M}\right)$ is said to satisfy the Dirac equation if $(i \not \nabla+\mu) h=0$.

Even though the Dirac operator is not normally hyperbolic it is possible to find unique advanced and retarded fundamental solutions on arbitrary globally hyperbolic spacetimes. The key element in this analysis is the Lichnérowicz identity,

$$
P=(-i \not \nabla+\mu)(i \not \nabla+\mu)
$$

where $P=\nabla^{2}+R / 4+\mu^{2}$ is the so-called supersymmetrically coupled Klein-Gordon operator for spinors, which relates Dirac operators to normally hyperbolic ones. The $R$ featuring in (16) is the Ricci scalar. It is known that there exist unique advanced $E_{P}^{-}$and retarded $E_{P}^{+}$fundamental solutions to any normally hyperbolic operator $P$ on globally hyperbolic spacetimes. Hence, for the spinor field, one has the following fundamental solutions: $S_{\mathrm{sp}}^{ \pm}=(i \not \nabla+\mu) E_{P}^{ \pm}$. To be explicit, $S_{\mathrm{sp}}^{ \pm}$are continuous operators $S_{\mathrm{sp}}^{ \pm}: C_{0}^{\infty}(D \mathcal{M}) \mapsto C^{\infty}(D \mathcal{M})$ such that

$$
(-i \not \nabla+\mu) S_{\mathrm{sp}}^{ \pm} f=S_{\mathrm{sp}}^{ \pm}(-i \not \nabla+\mu) f=f
$$

satisfying $\operatorname{supp}\left(S_{\mathrm{sp}}^{ \pm} f\right) \subset J^{ \pm}(\operatorname{supp} f)$. Clearly, there is a similar construction for the cospinor field resulting in $S_{\text {cosp }}^{ \pm}$. The advanced-minus-retarded fundamental solution for 
spinors is $S_{\mathrm{sp}}=S_{\mathrm{sp}}^{-}-S_{\mathrm{sp}}^{+}$and for cospinors $S_{\text {cosp }}=S_{\text {cosp }}^{-}-S_{\text {cosp }}^{+}$. An additional antilinear map $\Gamma: D^{*} \mathcal{M} \oplus D \mathcal{M} \mapsto D^{*} \mathcal{M} \oplus D \mathcal{M}$ acts by

$$
\Gamma\left(\begin{array}{c}
h \\
f
\end{array}\right)=\left(\begin{array}{l}
f^{+} \\
h^{+}
\end{array}\right)
$$

where $f \in C_{0}^{\infty}(D \mathcal{M})$ and $h \in C_{0}^{\infty}\left(D^{*} \mathcal{M}\right)$. The map $\Gamma$ makes explicit the symmetry between spinors and cospinors in this dual setting.

\subsection{The quantum Dirac field and Hadamard states}

To define the field algebra we denote by $\mathcal{D}\left(D^{*} \mathcal{M} \oplus D \mathcal{M}\right)=C_{0}^{\infty}\left(D^{*} \mathcal{M}\right) \oplus C_{0}^{\infty}(D \mathcal{M})$ the space of all test cospinors and test spinors on which the operators

$$
D:=i\left(\begin{array}{cc}
i \not \nabla+\mu & 0 \\
0 & -i \not \nabla+\mu
\end{array}\right) \quad S:=i\left(\begin{array}{cc}
S_{\operatorname{cosp}} & 0 \\
0 & S_{\mathrm{sp}}
\end{array}\right)
$$

act. The elements $\mathcal{F} \in \mathcal{D}\left(D^{*} \mathcal{M} \oplus D \mathcal{M}\right)$ may be used to label a set of abstract objects $\left\{\Psi(\mathcal{F}) \mid \mathcal{F} \in \mathcal{D}\left(D^{*} \mathcal{M} \oplus D \mathcal{M}\right)\right\}$ which, when equipped with $\mathbb{1}$, generates a unital *algebra $\mathfrak{F}$. We define the algebra of smeared fields $\mathfrak{F}(\mathcal{M}, g)$ to be $\mathfrak{F}$ quotiented by

i) Adjoint, $\Psi(\mathcal{F})^{*}=\Psi(\Gamma \mathcal{F})$;

ii) Linearity, $\Psi\left(\alpha_{1} \mathcal{F}_{1}+\alpha_{2} \mathcal{F}_{2}\right)=\alpha_{1} \Psi\left(\mathcal{F}_{1}\right)+\alpha_{2} \Psi\left(\mathcal{F}_{2}\right)$;

iii) The field equation, $\Psi(D \mathcal{F})=0$;

iv) Canonical anticommutation relation, $\left\{\Psi\left(\mathcal{F}_{1}\right), \Psi\left(\mathcal{F}_{2}\right)\right\}=i S\left(\mathcal{F}_{1}, \mathcal{F}_{2}\right) \mathbb{1}$.

Here $\mathcal{F}, \mathcal{F}_{1}, \mathcal{F}_{2} \in \mathcal{D}\left(D^{*} \mathcal{M} \oplus D \mathcal{M}\right)$ and $\alpha_{1}, \alpha_{2} \in \mathbb{C}$. It is relation (iv) that quantises the theory.

The usual Dirac field $\psi$ and its adjoint $\psi^{+}$are special cases of the above construction. For $h \in C_{0}^{\infty}\left(D^{*} \mathcal{M}\right), f \in C_{0}^{\infty}(D \mathcal{M})$ we define

$$
\psi(h):=\Psi\left(\begin{array}{c}
h \\
0
\end{array}\right) \quad \text { and } \quad \psi^{+}(f)=\Psi\left(\begin{array}{c}
0 \\
f
\end{array}\right)
$$

which we interpret as smeared fields.

A state $\omega: \mathfrak{F}(\mathcal{M}, g) \mapsto \mathbb{C}$ is a linear functional which is positive, i.e. $\omega\left(A^{*} A\right) \geq 0$ $\forall A \in \mathfrak{F}(\mathcal{M}, g)$, and normalised such that $\omega(\mathbb{1})=1$. We shall restrict our attention to states for which the two-point function, defined by

$$
\omega\left(\Psi\left(\mathcal{F}_{1}\right) \Psi\left(\mathcal{F}_{2}\right)\right) \quad \forall \mathcal{F}_{1}, \mathcal{F}_{2} \in \mathcal{D}\left(D^{*} \mathcal{M} \oplus D \mathcal{M}\right)
$$

is a distribution on $\mathcal{D}\left(D^{*} \mathcal{M} \oplus D \mathcal{M}\right) \otimes \mathcal{D}\left(D^{*} \mathcal{M} \oplus D \mathcal{M}\right)$. Associated to each state $\omega$ we define what we shall call the Dirac and Dirac adjoint two-point functions $\mathcal{W}_{\omega}, \mathcal{W}_{\omega}^{\Gamma} \in$ $\mathcal{D}^{\prime}\left(D \mathcal{M} \times D^{*} \mathcal{M}\right)$ respectively by

$$
\mathcal{W}_{\omega}(f, h)=\omega\left(\psi^{+}(f) \psi(h)\right) \text { and } \quad \mathcal{W}_{\omega}^{\Gamma}(f, h)=\omega\left(\psi(h) \psi^{+}(f)\right) .
$$


As a consquence of the positivity of states we immediately have that

$$
\mathcal{W}\left(f, f^{+}\right) \geq 0 \quad \forall f \in C_{0}^{\infty}(D \mathcal{M}) \quad \text { and } \quad \mathcal{W}^{\Gamma}\left(h^{+}, h\right) \geq 0 \quad \forall h \in C^{\infty}\left(D^{*} \mathcal{M}\right)
$$

The covariant anticommutation relation in terms of $\mathcal{W}_{\omega}$ and $\mathcal{W}_{\omega}^{\Gamma}$ is equally expressed

$$
\mathcal{W}_{\omega}+\mathcal{W}_{\omega}^{\Gamma}=i S_{\mathrm{sp}}
$$

It is clear from our discussion concerning the advanced and retarded fundamental solutions of the Dirac field that one may use the Lichnérowicz identity to define a notion of Hadamard state for the Dirac field. In order to give the precise formulation of the Hadamard series construction we must first discuss some geometry and here we follow [23]. We denote by $\mathfrak{X} \subset \mathcal{M} \times \mathcal{M}$ the set

$$
\begin{gathered}
\mathfrak{X}=\left\{\left(x, x^{\prime}\right) \in \mathcal{M} \times \mathcal{M} \mid x, x^{\prime}\right. \text { are causally related and } \\
J^{+}(x) \cap J^{-}\left(x^{\prime}\right) \text { and } J^{-}(x) \cap J^{+}\left(x^{\prime}\right) \text { are contained } \\
\text { within a convex normal neighbourhood }\} .
\end{gathered}
$$

Let $X \subset \mathfrak{X}$ be an open subset of $\mathfrak{X}$ such that between any pair $\left(x, x^{\prime}\right) \in X$ there exists a unique geodesic connecting them such that the (signed) geodesic separation of points defines a smooth function $\sigma$ on $X$. We make the additional requirement that the Hadamard construction (to be described shortly) can be carried out on $X$. Subject to all these requirements, we call $X$ a regular domain. We define two sequences of distributions $\left\{H_{k}^{( \pm)}\right\}_{k=0,1,2, \ldots} \in \mathcal{D}^{\prime}(X)$ by

$$
\begin{aligned}
H_{k}^{( \pm)}\left(x, x^{\prime}\right)=\frac{1}{4 \pi^{2}} & \left\{\frac{\Delta^{\frac{1}{2}}\left(x, x^{\prime}\right)}{\sigma_{ \pm}\left(x, x^{\prime}\right)}+\sum_{j=0}^{k} v_{j}\left(x, x^{\prime}\right) \frac{\sigma^{j}\left(x, x^{\prime}\right)}{\ell^{2(j+1)}} \ln \left(\frac{\sigma_{ \pm}\left(x, x^{\prime}\right)}{\ell^{2}}\right)\right. \\
& \left.+\sum_{j=0}^{k} w_{j}\left(x, x^{\prime}\right) \frac{\sigma^{j}\left(x, x^{\prime}\right)}{\ell^{2(j+1)}}\right\}
\end{aligned}
$$

where we have introduced a length scale $\ell$ to make $\sigma / \ell^{2}$ dimensionless. By $F\left(\sigma_{ \pm}\right), F$ some function, we mean

$$
F\left(\sigma_{ \pm}\right)=\lim _{\epsilon \rightarrow 0^{+}} F\left(\sigma_{ \pm \epsilon}\right)
$$

in the sense of distributions, where $\sigma_{ \pm \epsilon}\left(x, x^{\prime}\right)=\sigma\left(x, x^{\prime}\right) \pm 2 i \epsilon\left(t(x)-t\left(x^{\prime}\right)\right)+\epsilon^{2}$ and $t$ is a time function on $X$. The functions $\Delta$, known as the van Vleck-Morette determinant, $v_{j}$ and $w_{j}$ are found by fixing $x^{\prime}$ and applying $P \otimes \mathbb{1}$ to $H_{k}^{(+)}$and equating all the coefficients of $1 / \sigma_{+}, 1 / \sigma_{+}^{2}, \ln \sigma_{+}$etc to zero; moreover, they are all spinors (i.e., they carry internal indices). This determines a system of differential equations known as the Hadamard recursion relations 2 . In $X$ the system of differential equations uniquely determines the $\left\{v_{j}\right\}_{j=0, \ldots, k}$ series. The $\left\{w_{j}\right\}_{j=0, \ldots, k}$ series is specified once the value of $w_{0}$ is fixed; we adopt Wald's prescription that $w_{0}=0$ [27].

\footnotetext{
${ }^{2}$ The Hadamard recursions relations for the scalar field can be found in 12 .
} 
Let $u$ be of Hadamard form for the operator $P$, i.e. within a regular domain $X$ one has $u=H_{k}^{(+)}$modulo $C^{k}(X)$ for each $k \in \mathbb{N}$; the distribution $u$ is sometimes referred to as the auxiliary two-point function. A state $\omega$ on the algebra of smeared (Dirac) fields is said to be Hadamard if its associated two-point function $\mathcal{W}_{\omega}$ is of the form $\mathcal{W}_{\omega}=(i \not \nabla+\mu) \otimes \mathbb{1} u$ where $u$ is an auxiliary two-point function. Consequently, we have, within a regular domain $X$, that

$$
\mathcal{W}_{\omega}={ }^{\psi} H_{k}^{(+)} \text {modulo } C^{k}(X)
$$

where ${ }^{\psi} H_{k}^{(+)}=(i \not \nabla+\mu) \otimes \mathbb{1} H_{k+1}^{(+)}$. We also define ${ }^{\psi} H_{k}^{(-)}=(i \not \nabla+\mu) \otimes \mathbb{1} H_{k+1}^{(-)}$. Hence, within a regular domain $X$, for any Hadamard state $\omega$ we have the following identities:

$$
\begin{aligned}
\mathcal{W}_{\omega} & ={ }^{\psi} H_{k}^{(+)} \quad \text { modulo } C^{k}(X), \\
\mathcal{W}_{\omega}^{\Gamma} & =-{ }^{\psi} H_{k}^{(-)} \quad \text { modulo } C^{k}(X), \\
i S_{\mathrm{sp}} & ={ }^{\psi} H_{k}^{(+)}-{ }^{\psi} H_{k}^{(-)} \quad \text { modulo } C^{k}(X) .
\end{aligned}
$$

Two remarks are in order: First, note that we require the sign in (29) so as to ensure that the anticommutation relation holds. Second, observe that as $k$ increases $\mathcal{W}_{\omega}-{ }^{\psi} H_{k}^{(+)}$ becomes more regular and that, for sufficiently high $k, \mathcal{W}_{\omega}-{ }^{\psi} H_{k}^{(+)}$has a well defined coincidence limit.

\subsection{The stress energy tensor}

We open this section with a few remarks about obtaining scalar distributions from spinorial ones as this will be the basis of our analysis of the energy density for the remainder of our discussion. Let $E_{A}$ be the spinor field derived from a local section $E$ of the spin bundle $S \mathcal{M}$. Then one can derive matrices $\mathcal{W}_{\omega A B}$ and $\mathcal{W}_{\omega A B}^{\Gamma}$ from $\mathcal{W}_{\omega}$ and $\mathcal{W}_{\omega}^{\Gamma}$ via

$$
\mathcal{W}_{\omega A B}\left(f, f^{\prime}\right)=\mathcal{W}_{\omega}\left(f E_{A}, f^{\prime} E_{B}^{+}\right) \quad \mathcal{W}_{\omega A B}^{\Gamma}\left(f, f^{\prime}\right)=\mathcal{W}_{\omega}^{\Gamma}\left(f E_{A}, f^{\prime} E_{B}^{+}\right)
$$

for all $f, f^{\prime} \in C_{0}^{\infty}(\mathcal{M})$. Scalar bi-distributions $\mathrm{W}_{\omega}$ and $\mathrm{W}_{\omega}^{\Gamma}\left(\right.$ in $\mathcal{D}^{\prime}(\mathcal{M} \times \mathcal{M})$ ) may be constructed by taking the traces of the matrices, i.e. $\mathrm{W}_{\omega}=\delta^{A B} \mathcal{W}_{\omega A B}$ and $\mathrm{W}_{\omega}^{\Gamma}=$ $\delta^{A B} \mathcal{W}_{\omega A B}^{\Gamma}$. Similarly, one may define a scalar version ${ }^{\psi} \mathrm{H}_{k}^{( \pm)}$of the Hadamard series $H_{k}^{( \pm)}$.

The stress energy tensor of the classical spin- $1 / 2$ field $\psi$ is given by

$$
T_{a b}=\frac{i}{2}\left(\psi^{+} \gamma_{(a} \nabla_{b)} \psi-\left(\nabla_{(a} \psi^{+}\right) \gamma_{b)} \psi\right)
$$

where the subscript parentheses denote symmetrisation, i.e $\tau_{(a b)}=\left(\tau_{a b}+\tau_{b a}\right) / 2$. As advertised, we shall concentrate exclusively on proving an absolute QWEI along a timelike worldline. Therefore, we pick a properly parametrised smooth timelike worldline 
$\gamma: \mathbb{R} \mapsto \mathcal{M}$ and consider a spacetime tube $\tau_{\gamma} \subset \mathcal{M}$ centred about it, a precise construction of this tube will be given shortly. In $\tau_{\gamma}$ we may construct a tetrad $\left\{e_{\alpha}^{a}\right\}_{\alpha=0,1,2,3}$ such that $e_{0}^{a}=\dot{\gamma}^{a}$ and the remaining $\left\{e_{\alpha}^{a}\right\}_{\alpha=1,2,3}$ are orthonormal to this vector. Then, in the (dual) frame $e_{a}^{\alpha} \otimes e_{b}^{\beta}$, the tensor $T_{a b}$ has components $T_{\alpha \beta}$ given by

$$
T_{\alpha \beta}=\frac{i}{2}\left(\psi^{+} \gamma_{(\alpha} \nabla_{\beta)} \psi-\left(\nabla_{(\alpha} \psi^{+}\right) \gamma_{\beta)} \psi\right)
$$

from which follows the energy density $\rho$ :

$$
\rho=\frac{i}{2}\left(\psi^{+} \gamma_{0} \nabla_{0} \psi-\left(\nabla_{0} \psi^{+}\right) \gamma_{0} \psi\right)
$$

Recall from (10), that the spin frame $E_{A}$ and its Dirac conjugate satisfy $\delta^{A B} E_{A} \otimes$ $E_{B}^{+}=\gamma_{0}$. This enables us to write the classical point-split stress energy density $\rho^{\text {split }}$ of the Dirac field as

$$
\begin{aligned}
\rho^{\text {split }}\left(x, x^{\prime}\right)=\frac{i}{2} \delta^{A B} & \left(\left(\psi^{+} E_{A}\right) \otimes\left(E_{B}^{+} e_{0^{\prime}} \cdot \nabla \psi\right)\right. \\
& \left.-\left(\left[e_{0} \cdot \nabla \psi^{+}\right] E_{A}\right) \otimes\left(E_{B}^{+} \psi\right)\right)\left(x, x^{\prime}\right) .
\end{aligned}
$$

One may then use $\rho^{\text {split }}$ to define the distributional point-split energy density, also denoted $\rho^{\text {split: }}$ Let $f, f^{\prime} \in C_{0}^{\infty}(\mathcal{M})$ then

$$
\begin{aligned}
& \rho^{\mathrm{split}}\left(f^{\prime}, f\right)= \frac{i}{2} \delta^{A B} \int_{\mathcal{M} \times \mathcal{M}} \operatorname{dvol}(x) \operatorname{dvol}\left(x^{\prime}\right) \\
&\left(f^{\prime} \otimes f\right)\left(\left(\psi^{+} E_{A}\right) \otimes\left(E_{B}^{+} e_{0^{\prime}} \cdot \nabla \psi\right)\right. \\
&\left.\quad-\left(\left[e_{0} \cdot \nabla \psi^{+}\right] E_{A}\right) \otimes\left(E_{B}^{+} \psi\right)\right)\left(x, x^{\prime}\right) \\
&=\frac{i}{2} \delta^{A B}\left(\psi^{+}\left(f^{\prime} E_{A}\right) \psi\left(\nabla \cdot\left(e_{0^{\prime}} f E_{B}^{+}\right)\right)\right. \\
&\left.-\psi^{+}\left(\nabla \cdot\left(e_{0} f^{\prime} E_{A}\right)\right) \psi\left(f E_{B}^{+}\right)\right)
\end{aligned}
$$

where we have expressed the right hand side in more traditional distributional language and $u \nabla \cdot v$ denotes the distributional dual of $(\nabla u) \cdot v$. It is now clear that the replacement of $\psi^{+} \otimes \psi$ in (37) by the two-point function of any Hadamard state $\omega$ defines the expectation point-split energy density $\left\langle\rho^{\text {split }}\right\rangle_{\omega}$ of the Dirac field in that state. For notational convenience, we decompose $\left\langle\rho^{\text {split }}\right\rangle_{\omega}$ into the operator $\left(T_{00^{\prime}}^{\text {split }}\right)^{A B}$ acting on $\mathcal{W}_{\omega A B}-{ }^{\psi} H_{1 A B}^{(+)}$where $\left(T_{00^{\prime}}^{\text {split }}\right)^{A B}$ is given by

$$
\left(T_{00^{\prime}}^{\mathrm{split}}\right)^{A B}=\frac{i}{2} \delta^{A B}\left(\mathbb{1} \otimes e_{0} \cdot \nabla-e_{0} \cdot \nabla \otimes \mathbb{1}\right)+\Theta^{A B}
$$


and $\Theta^{A B}$ is a term which depends only on the spin-connection. The precise form of $\Theta^{A B}$ may be found in [7] (eqn. (3.10) of that reference) but does not affect our discussion due to a useful result 3 which shows it is identically zero on a timelike worldline under certain conditions which we shall now motivate: Let $\gamma: \mathbb{R} \mapsto \mathcal{M}$ be a timelike worldline with unit tangent vector $\dot{\gamma}$. Pick a point $x$ on $\gamma \subset \mathcal{M}$ and construct a local frame $\left\{e_{\alpha}^{a}\right\}_{\alpha=0,1,2,3}$ subject to $e_{0}^{a}=\dot{\gamma}^{a}$ at $x$. As we shall only be concerned with averaging along a compact subset of $\gamma$ we shall fix a closed interval $I \subset \gamma(\mathbb{R})$ such that $x \in I$. One may utilise Fermi-Walker transport to move $\left\{e_{\alpha}^{a}\right\}_{\alpha=0,1,2,3}$ along $I$ keeping $\left.e_{0}^{a}\right|_{\gamma}=\dot{\gamma}^{a}$. The salient feature of Fermi-Walker transport is that it preserves angles, i.e. $\left\{e_{\alpha}^{a}\right\}_{\alpha=0,1,2,3}$ remains an orthonormal family along $I$. Next, at each point $\gamma(s)$ along $I$ consider the convex normal neighbourhood $\mathcal{U}$ orthogonal to $\dot{\gamma}(s)$ and for each $y \in \mathcal{U}$ parallel transport $\left\{e_{\alpha}^{a}\right\}_{\alpha=0,1,2,3}$ along the unique geodesic connecting $y$ to $\gamma(s)$. In this manner we 'sweep out' a tube $\tau_{\gamma} \subset \mathcal{M}$ in spacetime. Importantly, the local frame (throughout $\tau_{\gamma}$ ) is a local section of $F \mathcal{M}$ which may be identified with a smooth section $E$ of $S \mathcal{M}$. The details of this identification may be found in $\S 3$ of [7]. For our purposes it is sufficient to know that, as a consequence of this construction, the form of $\left(T_{00^{\prime}}^{\text {split }}\right)^{A B}$ simplifies when restricted to the diagonal. The precise statement, quoted from [3] (lem.4) is:

Lemma 2.1. If $E$ is any local section of $S \mathcal{M}$ obtained in the above fashion from the curve $\gamma$, then $\left.\Theta^{A B}\right|_{\gamma}=0$.

We shall assume that $E$ has been obtained in this way. Therefore, the finite contribution $\left\langle\rho^{\text {fin }}\right\rangle_{\omega}$ to the energy density is given by:

$$
\left\langle\rho^{\mathrm{fin}}\right\rangle_{\omega}(\gamma(t))=\frac{i}{2} \vartheta^{*}\left(\left(\mathbb{1} \otimes e_{0} \cdot \nabla-e_{0} \cdot \nabla \otimes \mathbb{1}\right)\left(\mathrm{W}_{\omega}-{ }^{\psi} \mathrm{H}_{1}^{(+)}\right)\right)(t, t)
$$

where $\vartheta=\gamma \otimes \gamma$. Finally, it may be argued in analogy with [7] that one may re-express $\left\langle\rho^{\mathrm{fin}}\right\rangle_{\omega}$ as

$$
\left\langle\rho^{\mathrm{fin}}\right\rangle_{\omega}(\gamma(t))=\frac{1}{2}(\mathbb{1} \otimes D-D \otimes \mathbb{1}) \vartheta^{*}\left(\mathrm{~W}_{\omega}-{ }^{\psi} \mathrm{H}_{1}^{(+)}\right)(t, t)
$$

where $D$ is the distributional dual to $-i \mathrm{~d} / \mathrm{d} t$.

\section{Microlocal analysis applied to quantum field the- ory}

\subsection{The Sobolev wave-front set}

Since the publication of Radzikowski's equivalence theorem [22] microlocal analysis, in particular Hörmander's concept of wave-front set, has been successfully applied to

\footnotetext{
${ }^{3}$ Lemma 4 of $[3]$.
} 
quantum field theory 4 . The proof of the most general QWEIs rely on microlocal analysis at various stages in their argument, e.g. [3, 6, 9]. A refined version of the usual (smooth) wave-front set, an exposition of which may be found in chapter VIII of [15], has already been employed in the proof of an absolute quantum energy inequality for the KleinGordon field [12]. We shall briefly review the necessary details of this refinement, known as the Sobolev wave-front set.

For $s \in \mathbb{R}$, the Sobolev space $H^{s}\left(\mathbb{R}^{n}\right)$ is the set of all tempered distributions $u$ such that $\widehat{u}$ is a measurable function and

$$
\|u\|_{H^{s}\left(\mathbb{R}^{n}\right)}^{2}=\int_{\mathbb{R}^{n}} \mathrm{~d}^{n} \xi\left(1+|\xi|^{2}\right)^{s}|\widehat{u}(\xi)|^{2}<\infty .
$$

It is clear that $\mathcal{S}\left(\mathbb{R}^{n}\right) \subset H^{s}\left(\mathbb{R}^{n}\right)$ for each $s \in \mathbb{R}$. Moreover, one may show that $\mathcal{S}\left(\mathbb{R}^{n}\right)$ is dense in $H^{s}\left(\mathbb{R}^{n}\right)$, see chapter $1 \S 3$ of [25] for a brief argument.

Sobolev space theory is usually introduced into the study of distributional solutions to partial differential equations by asking when such a solution is an honest function; this is the subject of the embedding theorems. We summarise the following useful properties of the $H^{s}$ spaces, the first of which is a relevant embedding theorem:

Proposition 3.1. The Sobolev spaces $H^{s}\left(\mathbb{R}^{n}\right)$ have the following properties:

i) Let $k \in\{0\} \cup \mathbb{N}$ and $s \in \mathbb{R}$ satisfy $s>k+n / 2$ then $H^{s}\left(\mathbb{R}^{n}\right) \subset C^{k}\left(\mathbb{R}^{n}\right)$ is a continuous embedding

ii) $\quad H^{s}\left(\mathbb{R}^{n}\right) \subset H^{s^{\prime}}\left(\mathbb{R}^{n}\right) \forall s \geq s^{\prime}$;

iii) if $u \in H^{s}\left(\mathbb{R}^{n}\right), f \in C^{k}\left(\mathbb{R}^{n}\right)$ and $D^{\alpha} f \in L^{\infty}\left(\mathbb{R}^{n}\right) \forall|\alpha| \leq k$, where $D$ is a partial derivative operator and $\alpha$ is a multi-index, then for all $|s| \leq k$ $u \mapsto f u$ is a bounded linear map of $H^{s}\left(\mathbb{R}^{n}\right)$ into $H^{s}\left(\mathbb{R}^{n}\right)$. In particular, $H^{s}\left(\mathbb{R}^{n}\right)$ is closed under multiplication by smooth functions.

The concept of the Sobolev wave-front set will give a concise way of saying what it means for a distribution to microlocally fail to be an element of a Sobolev space. For convenience, we adopt the notation that $\dot{T}^{*} \mathbb{R}^{n}$ (similarly $\dot{T} \mathbb{R}^{n}, \dot{T} \mathcal{M}$ etc) is the bundle $T^{*} \mathbb{R}^{n}\left(T \mathbb{R}^{n}, T \mathcal{M}\right.$, etc) with the zero section removed.

Definition. A distribution $u \in \mathcal{D}^{\prime}\left(\mathbb{R}^{n}\right)$ is said to be microlocally $H^{s}$ at $(x, \xi) \in \dot{T}^{*} \mathbb{R}^{n}$ if there exists an open cone $\Gamma \subset \mathbb{R}^{n} \backslash 0$ about $\xi$ and a smooth function $\varphi \in C_{0}^{\infty}\left(\mathbb{R}^{n}\right)$, $\varphi(x) \neq 0$, such that

$$
\int_{\Gamma} \mathrm{d}^{n} \zeta\left(1+|\zeta|^{2}\right)^{s}\left|[\varphi u]^{\wedge}(\zeta)\right|^{2}<\infty
$$

The Sobolev wave-front set $W F^{s}(u)$ of a distribution $u \in \mathcal{D}^{\prime}\left(\mathbb{R}^{n}\right)$ is the complement, in $\dot{T}^{*} \mathbb{R}^{n}$, of the set of all pairs $(x, \xi)$ at which $u$ is microlocally $H^{s}$.

\footnotetext{
${ }^{4}$ For a readable account of the general significance of microlocal analysis in quantum field theory the reader is directed to [28].
} 
To define the Sobolev wave-front set of a distribution on a manifold one works locally. Let $\mathcal{U}$ be an open patch of a manifold $\mathcal{M}$ with associated coordinate map $\kappa: \mathcal{U} \mapsto \mathbb{R}^{n}$. If $(\kappa(x), \xi) \in W F^{s}\left(u \circ \kappa^{-1}\right)$ then $\left(x, \kappa_{*}^{-1}(\xi)\right) \in W F^{s}(u) \subset \dot{T}^{*} \mathcal{M}$. We shall occasionally use the notation $u \in H_{\text {loc }}^{s}(\mathcal{M})$ if $W F^{s}(u)=\emptyset$ for a distribution $u \in \mathcal{D}^{\prime}(\mathcal{M})$ and direct the reader to the remarks following definition 8.2.5 of [16] to justify this notation.

The Sobolev wave-front set is a closed cone in $\dot{T}^{*} \mathcal{M}$. Furthermore, we have the following properties 5 of $W F^{s}$ :

i) The smooth wave-front set is related to the Sobolev wave-front set

$$
\text { via } W F(u)=\overline{\bigcup_{s \in \mathbb{R}} W F^{s}(u)} \text {. }
$$

ii) If $\varphi \in C_{0}^{\infty}\left(\mathbb{R}^{n}\right)$ does not vanish in a neighbourhood of $x$ then $(x, \xi) \in W F^{s}(u)$ if and only if $(x, \xi) \in W F^{s}(\varphi u)$.

iii) $(x, \xi) \in W F^{s}(u)$ if and only if, for all $v \in H_{\mathrm{loc}}^{s},(x, \xi) \in W F^{s}(u-v)$.

iv) $W F^{s}(u+w) \subset W F^{s}(u) \cup W F^{s}(w)$.

v) The nesting property: $W F^{s}(u) \subset W F^{s^{\prime}}(u) \forall s \leq s^{\prime}$.

It is also possible to see explicitly in $W F^{s}$ what effect partial differential operators have on the singularities of distributions. For a general $m$-dimensional smooth manifold $\mathcal{M}$, let $P$ be a partial differential operator of order $r$, i.e. in local coordinates on $\mathcal{M}$

$$
P=\sum_{|\alpha| \leq r} p_{\alpha}(x)\left(-i \partial_{a}\right)^{\alpha}
$$

where $\alpha$ is a multi-index and $p_{\alpha}$ are smooth functions, then the principal symbol, $p_{r}(x, \xi)$, of $P$ is

$$
p_{r}(x, \xi)=\sum_{|\alpha|=r} p_{\alpha}(x) \xi_{a}^{\alpha} .
$$

The characteristic set, Char $P$, of a partial differential operator $P$ is the set of $(x, \xi) \in$ $\dot{T}^{*} \mathcal{M}$ such that the principal symbol vanishes. We may now quote corollaries $8.4 .9-10$ of [16] which show the effect differential operators have on the Sobolev wave-front set of a distribution:

Lemma 3.2. Let $\mathcal{M}$ be a smooth manifold. For $u \in \mathcal{D}^{\prime}(\mathcal{M})$ and any linear partial differential operator $P$ of order $r$ with smooth coefficients then $W F^{s}(P u) \subset W F^{s+r}(u)$ and $W F^{s+r}(u) \subset W F^{s}(P u) \cup$ Char $P$.

We close this section with the statement of Beal's restriction theorem, which tells us under what circumstances a distribution may be restricted to a submanifold, and a result about the implications for the positivity of states under such a restriction. Such results are of interest to us as we need to understand how to restrict those distributions which make up the Hadamard series (and ones derived from it such as the point-split energy density) to timelike worldlines.

Beal's restriction theorem tells us that, for certain well behaved restrictions, the Sobolev grading on the wave-front set is reduced by an amount proportional to the

\footnotetext{
${ }^{5}$ Taken from the remarks following definition B.1 of [17.
} 
codimension of the restriction. The result discusses the case of restricting a distribution on a $m$ dimensional manifold $\mathcal{M}$ to a smoothly embedded submanifold $\Sigma$ of dimension $n$, writing the embedding as $\iota: \Sigma \rightarrow \mathcal{M}$. The embedding function $\iota$ has associated conormal bundle $N^{*} \Sigma$ given by

$$
N^{*} \Sigma=\left\{(\iota(x), \xi) \in T^{*} \mathcal{M} ; x \in \Sigma, \iota^{*}(\xi)=0\right\} .
$$

We wish to formulate a statement of the restriction theorem for product manifolds 6 . As usual we let $(\mathcal{M}, g)$ denote a smooth $m$ dimensional spacetime, $\Sigma \subset \mathcal{M}$ an $n \leq m$ dimensional submanifold embedded using $\iota: \Sigma \mapsto \mathcal{M}$. Then we define the map $\vartheta$ : $\Sigma \times \Sigma \mapsto \mathcal{M} \times \mathcal{M}$ by $\vartheta=\iota \otimes \iota$, the pull back $\vartheta^{*}$ may sometimes be referred to as a restriction map.

Theorem 3.3 (Beal's Restriction theorem). Let $u \in \mathcal{D}^{\prime}(\mathcal{M} \times \mathcal{M})$ and $\vartheta$ be defined as above. If $\left(N^{*} \Sigma \times N^{*} \Sigma\right) \cap W F^{s}(u)=\emptyset$ for some $s>m-n$ then the restriction $\vartheta^{*} u$ of $u$ to $\Sigma \times \Sigma$ is a well defined distribution in $\mathcal{D}^{\prime}(\Sigma \times \Sigma)$. Moreover,

$$
W F^{s-(m-n)}\left(\vartheta^{*} u\right) \subset \vartheta^{*} W F^{s}(u)
$$

where the set $\vartheta^{*} W F^{s}(u)$ is defined to be

$$
\begin{array}{r}
\vartheta^{*} W F^{s}(u)=\left\{\left(t, \iota^{*}(\xi) ; t^{\prime}, \iota^{*}\left(\xi^{\prime}\right)\right) \in\left(T^{*} \Sigma \times T^{*} \Sigma\right) \mid\right. \\
\left.\left(\iota(t), \xi ; \iota\left(t^{\prime}\right), \xi^{\prime}\right) \in W F^{s}(u)\right\} .
\end{array}
$$

Finally, we state a result 7 which asserts that the positivity of states is preserved under the restrictions carried out by Beal's theorem.

Lemma 3.4. Let $\mathcal{M}$ and $\Sigma$ be smooth manifolds each equipped with smooth positive densities, and suppose $\iota: \Sigma \mapsto \mathcal{M}$ is smooth. If $u \in \mathcal{D}^{\prime}(\mathcal{M} \times \mathcal{M})$ is positive in the sense of states and $W F(u) \cap\left(N^{*} \Sigma \times N^{*} \Sigma\right)=\emptyset$, then $\vartheta^{*} u=u \circ(\iota \otimes \iota) \in \mathcal{D}^{\prime}(\Sigma \times \Sigma)$ is also positive.

\subsection{A microlocal description of the Hadamard series}

Denote by $\mathcal{R}=\left\{(x, \xi) \in \dot{T}^{*} \mathcal{M} \mid g^{a b}(x) \xi_{a} \xi_{b}=0\right\}$ the bundle of null covectors over $\mathcal{M}$. Since $(\mathcal{M}, g)$ is time orientable we may decompose $\mathcal{R}$ into two disjoint sets $\mathcal{R}^{ \pm}$defined by $\mathcal{R}^{ \pm}=\{(x, \xi) \in \mathcal{R} \mid \pm \xi \triangleright 0\}$ where by $\xi \triangleright 0\left(\xi \in T_{x}^{*} \mathcal{M}\right)$ we mean that $\xi_{a}$ is in the dual of the future light cone at $x$. We define the notation $(x, \xi) \sim\left(x^{\prime}, \xi^{\prime}\right)$ to mean that there exists a null curve $\gamma:[0,1] \mapsto \mathcal{M}$ such that $\gamma(0)=x, \gamma(1)=x^{\prime}$ and $\xi_{a}=\dot{\gamma}^{b}(0) g_{a b}(x)$,

\footnotetext{
${ }^{6}$ The following result is adapted from lemma 11.6.1 of [16] which is a refinement of the standard restriction theorem which may be found presented as theorem 8.2.4 of [15]. We have used the notation of prop. B7 of [17.

${ }^{7}$ Theorem 2.2 of $[6]$.
} 
$\xi_{a}^{\prime}=\dot{\gamma}^{b}(1) g_{a b}\left(x^{\prime}\right)$. In the instance where $x=x^{\prime},(x, \xi) \sim\left(x, \xi^{\prime}\right)$ shall mean that $\xi=\xi^{\prime}$ is null. Then, for convenience, define the set

$$
C=\left\{\left(x, \xi ; x^{\prime}, \xi^{\prime}\right) \in \mathcal{R} \times \mathcal{R} \mid(x, \xi) \sim\left(x^{\prime}, \xi^{\prime}\right)\right\} .
$$

The set $C^{+-}$is defined to be

$$
C^{+-}=\left\{\left(x, \xi ; x^{\prime},-\xi^{\prime}\right) \in C \mid \xi \triangleright 0\right\} .
$$

An occasionally useful set will be $C^{-+}$defined by

$$
C^{-+}=\left\{\left(x,-\xi ; x^{\prime}, \xi^{\prime}\right) \in C \mid \xi \triangleright 0\right\} .
$$

Junker \& Schrohe [17] have proven that the quantum Klein-Gordon field, whose two-point function we denote $\Lambda_{\omega}$, obeys the following condition for all Hadamard states

$$
W F^{s}\left(\Lambda_{\omega}\right)=\left\{\begin{array}{cc}
C^{+-} & s \geq-1 / 2 \\
\emptyset & s<-1 / 2
\end{array} .\right.
$$

In [12] an analysis of the Hadamard series $H_{k}^{(+)}$was given and concluded that

$$
W F^{s+j+1}\left(\sigma^{j} \ln \sigma_{+}\right) \subset W F^{s}\left(1 / \sigma_{+}\right)=\left\{\begin{array}{cc}
C^{+-} & s \geq-1 / 2 \\
\emptyset & s<-1 / 2
\end{array}\right.
$$

for $j \in\{0\} \cup \mathbb{N}$ and where $1 / \sigma_{+}$and $\sigma^{j} \ln \sigma_{+}$are the singular constituents of the Hadamard series $H_{k}^{(+)}(\underline{25})$. Since it is known that the the coefficients $\Delta^{\frac{1}{2}}$ and $v_{j}$ appearing in the $H_{k}^{(+)}$series are symmetric it follows from the simple symmetry argument $H_{k}^{(+)}\left(x, x^{\prime}\right)=H_{k}^{(-)}\left(x^{\prime}, x\right)$ modulo smooth functions that

$$
W F^{s+j+1}\left(\sigma^{j} \ln \sigma_{ \pm}\right) \subset W F^{s}\left(1 / \sigma_{ \pm}\right)=\left\{\begin{array}{cc}
C^{ \pm \mp} & s \geq-1 / 2 \\
\emptyset & s<-1 / 2
\end{array} .\right.
$$

Therefore, lemma 3.2 implies that if $\omega$ is a Hadamard state for the Dirac field then within a regular domain

$$
W F^{s}\left(\mathcal{W}_{\omega}\right) \subset\left\{\begin{array}{cc}
C^{+-} & s \geq 1 / 2 \\
\emptyset & s<1 / 2
\end{array}\right.
$$

A similar condition holds for $\mathcal{W}_{\omega}^{\Gamma}$ under the replacement $\mathcal{W}_{\omega} \mapsto \mathcal{W}_{\omega}^{\Gamma}$ and $C^{+-} \mapsto C^{-+}$ Moreover, one may use the relations (28,30) to conclude that within a regular domain

$$
\begin{aligned}
& W F^{s}\left(\mathcal{W}_{\omega}-{ }^{\psi} H_{k}^{(+)}\right) \subset\left\{\begin{array}{cc}
C^{+-} & s \geq k+3 / 2 \\
\emptyset & s<k+3 / 2
\end{array},\right. \\
& W F^{s}\left(\mathcal{W}_{\omega}^{\Gamma}-{ }^{\psi} H_{k}^{(-)}\right) \subset\left\{\begin{array}{cc}
C^{-+} & s \geq k+3 / 2 \\
\emptyset & s<k+3 / 2
\end{array},\right.
\end{aligned}
$$

where $\omega$ is a Hadamard state. Consequently, if $\omega$ is a Hadamard state then (because $\mathrm{W}_{\omega}, \mathrm{W}_{\omega}^{\Gamma}$ are formed from linear combinations of $\mathcal{W}_{\omega}$ and $\left.\mathcal{W}_{\omega}^{\Gamma}\right)$ it follows that the Sobolev wave-front set conditions which apply to $\mathcal{W}_{\omega}$ and $\mathcal{W}_{\omega}^{\Gamma}$ also apply to $\mathrm{W}_{\omega}$ and $\mathrm{W}_{\omega}^{\Gamma}$ respectively. We encapsulate these findings in the following corollary: 
Corollary 3.5. Let $\omega$ be a Hadamard state for the Dirac field; then within a regular domain

$$
\begin{aligned}
& W F^{s}\left(\mathrm{~W}_{\omega}-{ }^{\psi} \mathrm{H}_{k}^{(+)}\right) \subset\left\{\begin{array}{cc}
C^{+-} & s \geq k+3 / 2 \\
\emptyset & s<k+3 / 2
\end{array},\right. \\
& W F^{s}\left(\mathrm{~W}_{\omega}^{\Gamma}-{ }^{\psi} \mathrm{H}_{k}^{(-)}\right) \subset\left\{\begin{array}{cc}
C^{-+} & s \geq k+3 / 2 \\
\emptyset & s<k+3 / 2
\end{array} .\right.
\end{aligned}
$$

\section{A Sobolev point-splitting result}

We quote a result, taken from [7], for smooth functions:

Lemma 4.1. If $f \in C_{0}^{\infty}(\mathbb{R})$ and $u \in C_{0}^{\infty}(\mathbb{R} \times \mathbb{R})$ then the following identity holds:

$$
\int_{\mathbb{R}} \mathrm{d} t f^{2}(t) u(t, t)=\int_{\mathbb{R} \times \mathbb{R}} \frac{\mathrm{d} \xi \mathrm{d} \xi^{\prime}}{(2 \pi)^{2}} \widehat{f}^{2}\left(\xi-\xi^{\prime}\right) \hat{u}\left(-\xi, \xi^{\prime}\right) .
$$

This result forms the basis of the analysis in [7, 3] where the authors relate $u$ to the energy density obtained from normal ordering, i.e.

$$
\frac{1}{2}(\mathbb{1} \otimes D-D \otimes \mathbb{1}) \vartheta^{*}\left(\mathrm{~W}_{\omega}-\mathrm{W}_{\omega_{0}}\right)(t, t)
$$

where $\omega_{0}$ is another Hadamard state of the Dirac field. Since the difference between any two two-point functions arising from Hadamard states is smooth the quantity (60) is readily identifiable with $u$ in the hypothesis of lemma 4.1. However, $\mathrm{W}_{\omega}-{ }^{\psi} \mathrm{H}_{1}^{(+)}$ featuring in (40) is not smooth so we need to relax the hypothesis of lemma 4.1 in order to proceed. In particular we wish to show that one has the conclusion of lemma 4.1 under the weaker assumption that $u \in H^{s}(\mathbb{R} \times \mathbb{R}) \cap \mathcal{E}^{\prime}(\mathbb{R} \times \mathbb{R})$ for $s>1$. Let $u \in C_{0}^{\infty}(\mathbb{R} \times \mathbb{R})$; then, applying the Hölder inequality we have

$$
\begin{aligned}
\int_{\mathbb{R}} \mathrm{d} t\left|f^{2}(t) u(t, t)\right| & \leq\left\|f^{2}\right\|_{L^{1}(\mathbb{R})} \sup _{t \in \mathbb{R}}|u(t, t)| \\
& \leq\left\|f^{2}\right\|_{L^{1}(\mathbb{R})}\|u\|_{L^{\infty}(\mathbb{R} \times \mathbb{R})}
\end{aligned}
$$

for all $u \in C_{0}^{\infty}(\mathbb{R} \times \mathbb{R})$; we also remark that $\|\cdot\|_{L^{\infty}(\mathbb{R} \times \mathbb{R})}$ is the natural norm on $C(\mathbb{R} \times \mathbb{R})$. Since the embedding of $H^{s}(\mathbb{R} \times \mathbb{R})$ into $C(\mathbb{R} \times \mathbb{R})$ is continuous for $s>1$ there exists a constant $c>0$ such that $\|u\|_{L^{\infty}(\mathbb{R} \times \mathbb{R})} \leq c\|u\|_{H^{s}(\mathbb{R} \times \mathbb{R})}$ for all $u \in H^{s}(\mathbb{R} \times \mathbb{R})$ and $s>1$. Hence,

$$
\int_{\mathbb{R}} \mathrm{d} t\left|f^{2}(t) u(t, t)\right| \leq c\left\|f^{2}\right\|_{L^{1}(\mathbb{R})}\|u\|_{H^{s}(\mathbb{R} \times \mathbb{R})} \quad \forall u \in C_{0}^{\infty}(\mathbb{R} \times \mathbb{R}) \quad s>1 .
$$

Moreover, as $C_{0}^{\infty}(\mathbb{R} \times \mathbb{R})$ is dense in $H^{s}(\mathbb{R} \times \mathbb{R})$, the Bounded Linear Transform theorem implies that (63) holds for all $u \in H^{s}(\mathbb{R} \times \mathbb{R})$ for $s>1$. Equally, we may apply the Hölder inequality to the right-hand-side of $(59)$ to obtain

$$
\left|\int_{\mathbb{R} \times \mathbb{R}} \frac{\mathrm{d} \xi \mathrm{d} \xi^{\prime}}{(2 \pi)^{2}} \widehat{f}^{2}\left(\xi-\xi^{\prime}\right) \widehat{u}\left(-\xi, \xi^{\prime}\right)\right| \leq\|F\|_{L^{\infty}(\mathbb{R} \times \mathbb{R})}\|\widehat{u}\|_{L^{1}(\mathbb{R} \times \mathbb{R})},
$$


where $F\left(\xi, \xi^{\prime}\right)=\widehat{f^{2}}\left(\xi-\xi^{\prime}\right)$. A factor of $\left(1+|\xi|^{2}+\left|\xi^{\prime}\right|^{2}\right)^{(1+\varepsilon) / 2}\left(1+|\xi|^{2}+\left|\xi^{\prime}\right|^{2}\right)^{-(1+\varepsilon) / 2}$ is then introduced into the $L^{1}$ norm and the Cauchy-Schwarz inequality applied to obtain

$$
\|\widehat{u}\|_{L^{1}(\mathbb{R} \times \mathbb{R})} \leq \sqrt{\frac{\pi}{\epsilon}}\|u\|_{H^{1+\varepsilon}(\mathbb{R} \times \mathbb{R})}
$$

where we have written $s>1$ as $s=1+\epsilon$. Again the Bounded Linear Transform theorem implies that (65) holds for all $u \in H^{1+\epsilon}(\mathbb{R} \times \mathbb{R})$ where the ${ }^{-}$now refers to the continuous extension of the Fourier transform to $H^{1+\epsilon}(\mathbb{R} \times \mathbb{R}$ ) (although this must agree with the usual Fourier transform on $L^{2}(\mathbb{R} \times \mathbb{R})$ or $\left.\mathcal{S}^{\prime}(\mathbb{R} \times \mathbb{R})\right)$. Therefore, since (59) holds for a dense subset of $H^{1+\epsilon}(\mathbb{R} \times \mathbb{R})$ and may be extended continuously onto the whole of the space, we have proven:

Lemma 4.2. Let $f \in C_{0}^{\infty}(\mathbb{R})$ and $u \in H^{s}(\mathbb{R} \times \mathbb{R}) \cap \mathcal{E}^{\prime}(\mathbb{R} \times \mathbb{R}), s>1$, then the following identity holds:

$$
\int_{\mathbb{R}} \mathrm{d} t f^{2}(t) u(t, t)=\int_{\mathbb{R} \times \mathbb{R}} \frac{\mathrm{d} \xi \mathrm{d} \xi^{\prime}}{(2 \pi)^{2}} \widehat{f}^{2}\left(\xi-\xi^{\prime}\right) \hat{u}\left(-\xi, \xi^{\prime}\right)
$$

It is clear under the identification $u\left(t, t^{\prime}\right)=\left\langle\rho^{\mathrm{fin}}\right\rangle_{\omega}(\gamma(t))$ (cf. (40) $)$ that

$$
\begin{aligned}
& \int_{\mathbb{R}} \mathrm{d} t f^{2}(t)\left\langle\rho^{\mathrm{fin}}\right\rangle_{\omega}(\gamma(t)) \\
& =\frac{1}{2} \int_{\mathbb{R} \times \mathbb{R}} \frac{\mathrm{d} \xi \mathrm{d} \xi^{\prime}}{(2 \pi)^{2}}\left(\xi+\xi^{\prime}\right) \widehat{f^{2}}\left(\xi-\xi^{\prime}\right)\left[\vartheta^{*}\left(\mathrm{~W}_{\omega}-{ }^{\psi} \mathrm{H}_{1}^{(+)}\right)\right]^{\wedge}\left(-\xi, \xi^{\prime}\right) .
\end{aligned}
$$

We now prove a result (similar to lemma 5 of [3] in all but one detail of the proo 8 ) which will enable us to relate the right hand side of (67) to an integral over the diagonal:

Lemma 4.3. If $f \in C_{0}^{\infty}(\mathbb{R})$ is real valued and $u \in H^{s}(\mathbb{R} \times \mathbb{R}), s>2$, is compactly supported then

$$
\int_{\mathbb{R} \times \mathbb{R}} \frac{\mathrm{d} \xi \mathrm{d} \xi^{\prime}}{(2 \pi)^{2}}\left(\xi+\xi^{\prime}\right) \widehat{f}^{2}\left(\xi-\xi^{\prime}\right) \hat{u}\left(-\xi, \xi^{\prime}\right)=\frac{1}{\pi} \int_{\mathbb{R}} \mathrm{d} \xi \xi u\left(\overline{f^{\xi}}, f^{\xi}\right)
$$

where $f^{\xi}(t)=e^{i \xi t} f(t)$.

Proof. Lemma 6.1 of [7] states that

$$
\left(\xi+\xi^{\prime}\right) \widehat{f}^{2}\left(\xi-\xi^{\prime}\right)=\frac{1}{\pi} \int_{\mathbb{R}} \mathrm{d} \zeta \zeta \hat{f}(\xi-\zeta) \overline{\hat{f}\left(\xi^{\prime}-\zeta\right)}
$$

and therefore

$$
\begin{aligned}
& \int_{\mathbb{R} \times \mathbb{R}} \frac{\mathrm{d} \xi \mathrm{d} \xi^{\prime}}{(2 \pi)^{2}}\left(\xi+\xi^{\prime}\right) \hat{f}^{2}\left(\xi-\xi^{\prime}\right) \hat{u}\left(-\xi, \xi^{\prime}\right) \\
& \quad=\frac{1}{\pi} \int_{\mathbb{R} \times \mathbb{R}} \frac{\mathrm{d} \xi \mathrm{d} \xi^{\prime}}{(2 \pi)^{2}} \int_{\mathbb{R}} \mathrm{d} \zeta \zeta \hat{f}(\xi-\zeta) \overline{\hat{f}\left(\xi^{\prime}-\zeta\right)} \hat{u}\left(-\xi, \xi^{\prime}\right) .
\end{aligned}
$$

\footnotetext{
${ }^{8}$ The distinct step is contained within line (79).
} 
We also note, by a simple application of the convolution theorem, that

$$
\begin{aligned}
u\left(\overline{f^{\xi}}, f^{\xi}\right) & =[(f \otimes f) u]\left(e^{-i \xi}, e^{i \xi \cdot}\right) \\
& =\int_{\mathbb{R} \times \mathbb{R}} \frac{\mathrm{d} \zeta \mathrm{d} \zeta^{\prime}}{(2 \pi)^{2}} \hat{f}(-\xi-\zeta) \hat{f}\left(\xi-\zeta^{\prime}\right) \hat{u}\left(\zeta, \zeta^{\prime}\right) \\
& =\int_{\mathbb{R} \times \mathbb{R}} \frac{\mathrm{d} \zeta \mathrm{d} \zeta^{\prime}}{(2 \pi)^{2}} \hat{f}(-\xi+\zeta) \hat{f}\left(\xi-\zeta^{\prime}\right) \hat{u}\left(-\zeta, \zeta^{\prime}\right) \\
& =\int_{\mathbb{R} \times \mathbb{R}} \frac{\mathrm{d} \zeta \mathrm{d} \zeta^{\prime}}{(2 \pi)^{2}} \hat{f}(\zeta-\xi) \overline{\hat{f}\left(\zeta^{\prime}-\xi\right)} \hat{u}\left(-\zeta, \zeta^{\prime}\right)
\end{aligned}
$$

and, therefore, that the statement of the theorem will be established if the integrals in (170) can be reordered. If we estimate $|\hat{f}(x)| \leq c /\left(1+|x|^{2}\right)$ then, by the arithmeticgeometric mean inequality

$$
\begin{aligned}
\int_{\mathbb{R}} \mathrm{d} \zeta & \left|\zeta \hat{f}(\xi-\zeta) \overline{\hat{f}\left(\xi^{\prime}-\zeta\right)}\right| \\
& \leq \frac{c^{2}}{2} \int_{\mathbb{R}} \mathrm{d} \zeta\left(\frac{|\zeta|}{\left(1+|\xi-\zeta|^{2}\right)^{2}}+\frac{|\zeta|}{\left(1+\left|\xi^{\prime}-\zeta\right|^{2}\right)^{2}}\right) \\
& \leq \frac{c^{2}}{2}\left(2+|\xi \arctan \xi|+\left|\xi^{\prime} \arctan \xi^{\prime}\right|\right) \\
& \leq \frac{c^{2} \pi}{4}\left(2+|\xi|+\left|\xi^{\prime}\right|\right) .
\end{aligned}
$$

Then

$$
\begin{aligned}
& \frac{1}{\pi} \int_{\mathbb{R} \times \mathbb{R} \times \mathbb{R}} \frac{\mathrm{d} \xi \mathrm{d} \xi^{\prime}}{(2 \pi)^{2}} \mathrm{~d} \zeta\left|\zeta \hat{f}(\xi-\zeta) \overline{\hat{f}\left(\xi^{\prime}-\zeta\right)} \hat{u}\left(-\xi, \xi^{\prime}\right)\right| \\
& \quad \leq \frac{c^{2}}{4} \int_{\mathbb{R} \times \mathbb{R}} \frac{\mathrm{d} \xi \mathrm{d} \xi^{\prime}}{(2 \pi)^{2}}\left(2+|\xi|+\left|\xi^{\prime}\right|\right)\left|\hat{u}\left(-\xi, \xi^{\prime}\right)\right| \\
& \quad \leq \frac{c^{2}}{16 \pi^{2}}\left\|p_{s}\right\|_{L^{2}(\mathbb{R} \times \mathbb{R})}\|u\|_{H^{s}(\mathbb{R} \times \mathbb{R})}
\end{aligned}
$$

where we have used the Cauchy-Schwarz inequality and written

$$
p_{s}\left(\xi, \xi^{\prime}\right)=\frac{2+|\xi|+\left|\xi^{\prime}\right|}{\left(1+|\xi|^{2}+\left|\xi^{\prime}\right|^{2}\right)^{s / 2}} \text {. }
$$

The $L^{1}$ norm of $\zeta \hat{f}(\xi-\zeta) \overline{\hat{f}\left(\xi^{\prime}-\zeta\right)} \hat{u}\left(-\xi, \xi^{\prime}\right)$ will be finite if $u \in H^{s}(\mathbb{R} \times \mathbb{R})$ and $s>2$. Under these conditions, Fubini's theorem implies that the integrals can be reordered to obtain the desired result.

\section{A worldline absolute quantum weak energy in- equality}

We are now in a position to state our result concerning the Dirac field. 
Theorem 5.1. Let $\omega$ be a Hadamard state for the Dirac field, $\gamma$ be a timelike worldline and $f \in C_{0}^{\infty}(\mathbb{R})$ be real valued; then

$$
\int_{\mathbb{R}} \mathrm{d} t f^{2}(t)\left\langle\rho^{\mathrm{fin}}\right\rangle_{\omega}(\gamma(t)) \geq-B
$$

where

$$
\begin{aligned}
B= & \int_{\mathbb{R}^{+}} \frac{\mathrm{d} \xi}{2 \pi} \xi\left[f \otimes f \vartheta^{*} \psi \mathrm{H}_{4}^{(+)}\right]^{\wedge}(-\xi, \xi) \\
& -\int_{\mathbb{R}^{-}} \frac{\mathrm{d} \xi}{2 \pi} \xi\left[f \otimes f \vartheta^{*}\left(i \mathrm{~S}_{\mathrm{sp}}-{ }^{\psi} \mathrm{H}_{4}^{(+)}\right)\right]^{\wedge}(-\xi, \xi)
\end{aligned}
$$

and $\vartheta=\gamma \otimes \gamma$.

Proof. Corollary 3.5 implies that $\mathrm{W}_{\omega}-{ }^{\psi} \mathrm{H}_{4}^{(+)} \in H_{\text {loc }}^{5+\varepsilon}(X)$ from which it follows that

$$
f \otimes f \vartheta^{*}\left(\left(\mathbb{1} \otimes i e_{0} \cdot \nabla-i e_{o} \cdot \nabla \otimes \mathbb{1}\right)\left(\mathrm{W}_{\omega}-{ }^{\psi} \mathrm{H}_{4}^{(+)}\right)\right) \in H_{\mathrm{loc}}^{1+\varepsilon}(\mathbb{R} \times \mathbb{R}),
$$

where we have lost one Sobolev order as a result of differentiation and a further three from the restriction to $\mathbb{R} \times \mathbb{R}$. Lemma 4.2, and the remarks following the proof, enable us to write

$$
\begin{aligned}
\int_{\mathbb{R}} \mathrm{d} t & f^{2}(t)\left\langle\rho^{\mathrm{fin}}\right\rangle_{\omega}(\gamma(t)) \\
= & \frac{1}{2} \int_{\mathbb{R} \times \mathbb{R}} \frac{\mathrm{d} \xi \mathrm{d} \xi^{\prime}}{(2 \pi)^{2}}\left(\xi+\xi^{\prime}\right) \widehat{f}^{2}\left(\xi-\xi^{\prime}\right)\left[\vartheta^{*}\left(\mathrm{~W}_{\omega}-{ }^{\psi} \mathrm{H}_{4}^{(+)}\right)\right]^{\wedge}\left(-\xi, \xi^{\prime}\right) .
\end{aligned}
$$

and as $\vartheta^{*}\left(\mathrm{~W}_{\omega}-{ }^{\psi} \mathrm{H}_{4}^{(+)}\right) \in H_{\text {loc }}^{2+\varepsilon}(\mathbb{R} \times \mathbb{R})$ we can employ lemma 4.3 to obtain:

$$
\int_{\mathbb{R}} \mathrm{d} t f^{2}(t)\left\langle\rho^{\mathrm{fin}}\right\rangle_{\omega}(t)=\int_{\mathbb{R}} \frac{\mathrm{d} \xi}{2 \pi} \xi \vartheta^{*}\left(\mathrm{~W}_{\omega}-{ }^{\psi} \mathrm{H}_{4}^{(+)}\right)\left(\overline{f^{\xi}}, f^{\xi}\right) .
$$

We decompose the integral into its positive and negative frequency components and appeal to the anticommutation relation (in scalar form) $\mathrm{W}_{\omega}+\mathrm{W}_{\omega}^{\Gamma}=i \mathrm{~S}_{\mathrm{sp}}$ to write

$$
\begin{aligned}
\int_{\mathbb{R}} \mathrm{d} t f^{2}(t)\left\langle\rho^{\mathrm{fin}}\right\rangle_{\omega}(t)=\int_{\mathbb{R}^{+}} & \frac{\mathrm{d} \xi}{2 \pi} \xi \vartheta^{*}\left(\mathrm{~W}_{\omega}-{ }^{\psi} \mathrm{H}_{4}^{(+)}\right)\left(\overline{f^{\xi}}, f^{\xi}\right) \\
& +\int_{\mathbb{R}^{-}} \frac{\mathrm{d} \xi}{2 \pi} \xi \vartheta^{*}\left(i \mathrm{~S}_{\mathrm{sp}}-\mathrm{W}_{\omega}^{\Gamma}-{ }^{\psi} \mathrm{H}_{4}^{(+)}\right)\left(\overline{f^{\xi}}, f^{\xi}\right) .
\end{aligned}
$$

Recall that $\mathrm{W}_{\omega}$ and $\mathrm{W}_{\omega}^{\Gamma}$ are distributions of positive type, hence

$$
\begin{aligned}
\int_{\mathbb{R}} \mathrm{d} t f^{2}(t)\left\langle\rho^{\mathrm{fin}}\right\rangle_{\omega}(t) \geq-\int_{\mathbb{R}^{+}} & \frac{\mathrm{d} \xi}{2 \pi} \xi \vartheta^{*} \psi \mathrm{H}_{4}^{(+)}\left(\overline{f^{\xi}}, f^{\xi}\right) \\
& +\int_{\mathbb{R}^{-}} \frac{\mathrm{d} \xi}{2 \pi} \xi \vartheta^{*}\left(i \mathrm{~S}_{\mathrm{sp}}-{ }^{\psi} \mathrm{H}_{4}^{(+)}\right)\left(\overline{f^{\xi}}, f^{\xi}\right)
\end{aligned}
$$


and it remains to show that this lower bound is finite.

We make the replacements of ${ }^{\psi} \mathrm{H}_{4}^{(+)}=\mathrm{W}_{\omega_{0}}-F$ and $i \mathrm{~S}_{\mathrm{sp}}-{ }^{\psi} \mathrm{H}_{4}^{(-)}=\mathrm{W}_{\omega_{0}}^{\Gamma}-G$ where $F, G \in C^{4}(X)$ and $\mathrm{W}_{\omega_{0}}, \mathrm{~W}_{\omega_{0}}^{\Gamma}$ arise from some arbitrary Hadamard state $\omega_{0}$. We remark that this replacement is a technical device only which we introduce to prove finiteness: The bound is still independent of any state. Hence, we have

$$
\begin{aligned}
B= & \int_{\mathbb{R}^{+}} \frac{\mathrm{d} \xi}{2 \pi} \xi\left[f \otimes f \vartheta^{*} \mathrm{~W}_{\omega_{0}}\right]^{\wedge}(-\xi, \xi)-\int_{\mathbb{R}^{-}} \frac{\mathrm{d} \xi}{2 \pi} \xi\left[f \otimes f \vartheta^{*} \mathrm{~W}_{\omega_{0}}^{\Gamma}\right]^{\wedge}(-\xi, \xi) \\
& -\int_{\mathbb{R}^{+}} \frac{\mathrm{d} \xi}{2 \pi} \xi\left[f \otimes f \vartheta^{*} F\right]^{\wedge}(-\xi, \xi)+\int_{\mathbb{R}^{-}} \frac{\mathrm{d} \xi}{2 \pi} \xi\left[f \otimes f \vartheta^{*} G\right]^{\wedge}(-\xi, \xi) .
\end{aligned}
$$

The finiteness of the $\mathrm{W}_{\omega_{0}}$ and $\mathrm{W}_{\omega_{0}}^{\Gamma}$ pieces is proven by the wave-front set conditions, $W F\left(\mathrm{~W}_{\omega_{0}}\right) \subset C^{+-}$and $W F\left(\mathrm{~W}_{\omega_{0}}^{\Gamma}\right) \subset C^{-+}$, which imply 9 that $\vartheta^{*} \mathrm{~W}_{\omega_{0}}$ and $\vartheta^{*} \mathrm{~W}_{\omega_{0}}^{\Gamma}$ are rapidly decaying in the directions they are being integrated in. Finally, one may use the following estimates

$$
\begin{aligned}
{\left[f \otimes f \vartheta^{*} F\right]^{\wedge}\left(\xi, \xi^{\prime}\right) } & \leq \frac{c}{\left(1+|\xi|^{2}+\left|\xi^{\prime}\right|^{2}\right)^{2}} \\
{\left[f \otimes f \vartheta^{*} G\right]^{\wedge}\left(\xi, \xi^{\prime}\right) } & \leq \frac{c^{\prime}}{\left(1+|\xi|^{2}+\left|\xi^{\prime}\right|^{2}\right)^{2}}
\end{aligned}
$$

Hence, our bound is finite.

Theorem 5.1 enables us to finally formulate our absolute QWEI for the Dirac field. Wald's uniqueness theorem implies that the regularised energy density $\left\langle\rho^{\text {fin }}\right\rangle_{\omega}$ we have computed is equal to the renormalised energy density $\left\langle\rho^{\text {ren }}\right\rangle_{\omega}$ up to the addition of a local curvature term $C$. Hence, our result reads:

$$
\int_{\mathbb{R}} \mathrm{d} t f^{2}(t)\left\langle\rho^{\mathrm{ren}}\right\rangle_{\omega}(t) \geq-\mathcal{B}
$$

where $\mathcal{B}$ is given by

$$
\begin{aligned}
\mathcal{B}=\int_{\mathbb{R}^{+}} & \frac{\mathrm{d} \xi}{2 \pi} \xi\left[f \otimes f \vartheta^{*} \psi \mathrm{H}_{4}^{(+)}\right]^{\wedge}(-\xi, \xi) \\
- & \int_{\mathbb{R}^{-}} \frac{\mathrm{d} \xi}{2 \pi} \xi\left[f \otimes f \vartheta^{*}\left(i \mathrm{~S}_{\mathrm{sp}}-{ }^{\psi} \mathrm{H}_{4}^{(+)}\right)\right]^{\wedge}(-\xi, \xi) \\
& -\int_{\mathbb{R}} \mathrm{d} t f^{2}(t) C(t)
\end{aligned}
$$

As reported in 12, where a more complete discussion of the renormalisation of the stress tensor of a quantum field may be found, the view may be held that the value of this curvature term (alongside the mass and curvature coupling) is an essential detail in

${ }^{9}$ For the full details of this step the reader is directed to $\S 3.2$ of [3]. 
the specification of the theory and that $C$ should be, at least in principle, measurable. Alternatively, one may hold the view that this unavoidable ambiguity is a manifestation of a breakdown of the semi-classical theory and that a more complete theory of quantum gravity is needed.

\section{Conclusion}

We have succeeded in proving a new absolute QWEI for the Dirac field under general circumstances. By exploiting a Sobolev graded refinement of Hörmander's wave-front set we have been able to modify the proof Fewster and Dawson [3] give for their difference QWEI and remove any reference to a state in the bound. Moreover, it is straightforward to use the techniques of [12] to obtain additional $W F^{s}$ information of the constituents of the Dirac Hadamard series ${ }^{\psi} H_{k}^{( \pm)}$.

\section{Acknowledgements}

The author would like to thank C.J. Fewster for his guidance over the course of this research. Additional thanks go to J.A. Sanders, L.W. Osterbrink, S.P. Dawson and P. Watts for their helpful comments on the manuscript.

\section{References}

[1] Alcubierre M., 1994, The warp drive: hyper-fast travel within general relativity, Class. Quantum Grav. 11, L73-L77

[2] Dawson S.P., 2006, A quantum weak energy inequality for the Dirac field in twodimensional flat spacetime, Class. Quantum Grav. 23, 287-293

[3] Dawson S.P. \& Fewster C.J., 2006, An explicit quantum weak energy inequality for Dirac fields in curved spacetimes, Class. Quantum Grav. 23, 6659-6681

[4] Dimock J., 1982, Dirac quantum fields on a manifold, Trans. Am. Math. Soc. Volume 232 No. 1 133-147

[5] Epstein H., Jaffe A. \& Glaser V., 1965, Nonpositivity in the energy density in quantised field theories, Nuovo Cimento 36, 1016-1022

[6] Fewster C.J., 2000, A general worldline quantum inequality, Class. Quantum Grav. 17, 1897-1911

[7] Fewster C.J. \& Verch R., 2002, A quantum weak energy inequality for Dirac fields in curved spacetime, Commun. Math. Phys. 225, 331-359

[8] Fewster C.J. \& Mistry B., 2003, Quantum weak energy inequalities for the Dirac field in flat spacetime, Phys. Rev. D 68, 105010 
[9] Fewster C.J. \& Pfenning M.J., 2003, A weak quantum energy inequality for spin-one fields in curved spacetime, J. Math. Phys. 44, 4480-4513

[10] Fewster C.J., 2005, Energy inequalities in quantum field theory, in XIVth International Congress on Mathematical Physics, ed. J.C. Zambrini (World Scientific, Singapore, 2005). See math-ph/0501073 for an expanded and updated version.

[11] Fewster C.J., 2005, Quantum energy inequalities and stability conditions in quantum field theory, in Rigorous Quantum Field Theory: A Festschrift for Jacques Bros, A. Boutet de Monvel, D. Buchholz, D. Iagolnitzer, U. Moschella (Eds.) Progress in Mathematics, Vol. 251. (Birkhäuser, Boston, 2006), math-ph/0502002.

[12] Fewster C.J. \& Smith C.J., 2007, Absolute quantum energy inequalities in curved spacetime, gr-qc/0702056

[13] Ford L.H., 1978, Quantum coherence effects and the second law of thermodynamics, Proc. R. Soc. Lond. A. 364, 227-236

[14] Hollands S., 2000, DPhil. thesis Aspects of quantum field theory in curved spacetime, Department of Mathematics, University of York, United Kingdom

[15] Hörmander L., 1989, The analysis of linear partial differential operators I second edition, Springer-Verlag, New York

[16] Hörmander L., 1996, Lectures on nonlinear hyperbolic differential equations, Springer, New York

[17] Junker W. \& Schrohe E., 2002, Adiabatic vacuum states on general spacetime manifolds: Definition, construction, and physical properties, Annales Poincaré Phys. Theor. 3, 1113-1182

[18] Kay B.S. \& Wald R.M., 1991, Theorems on the uniqueness and thermal properties of stationary, nonsingular, quasifree states on spacetimes with a bifurcate Killing horizon, Phys. Rep. 207, 49-136

[19] Köhler M., 1995, PhD. dissertation The stress energy tensor of a locally supersymmetric quantum field on a curved spacetime, gr-qc/9505014

[20] Kratzert K., 2000, Singularity structure of the two point function of the free Dirac field on globally hyperbolic spacetime, math-ph/0003015

[21] Nakahara M., 1989, Geometry, topology and physics, Institute of Physics publishing

[22] Radzikowski M., 1996, Micro-local approach to the Hadamard condition in quantum field theory on curved space-time, Commun. Math. Phys. 179, 529-553

[23] Sahlmann H. \& Verch R., 2001, Microlocal spectral condition and Hadamard form for vector-valued quantum fields in curved spacetime, Rev. Math. Phys. 13, 12031246

[24] Synge J., 1960, Relativity: The general theory, North Holland, Amsterdam

[25] Taylor M.E., 1981, Pseudodifferential operators, Princeton University Press, New Jersey

[26] Vollick D.N., 2000, Quantum inequalities in curved two dimensional spacetimes, Phys. Rev. D 61, 084022 
[27] Wald R.M., 1978, Trace anomaly of a conformally invariant quantum field in curved spacetime, Phys. Rev. D 17, 1477-1484

[28] Wald R.M., 2006, The history and present status of quantum field theory in curved spacetime, gr-qc/0608018 